\title{
RESIDUAL-FREE BUBBLE METHODS FOR NUMERICAL HOMOGENIZATION OF ELLIPTIC PROBLEMS*
}

\author{
TAO YU $\mathrm{YU}^{\dagger}$ AND XINGYE YUE
}

\begin{abstract}
The residual-free bubble (RFB) method was first proposed for convection-diffusion problems. Then it was used to treat elliptic homogenization problems. We revisit it in this paper and try to reveal its relations to some other multiscale methods. Some new bubble schemes are also proposed.
\end{abstract}

Key words. Residual-free bubble, numerical homogenization, multiscale finite element method, heterogeneous multiscale method, adaptive sampling.

AMS subject classifications. 65 N30.

\section{Introduction}

Consider the numerical homogenization of the following second order elliptic problems with oscillating data:

$$
\begin{aligned}
& \mathbb{L} u_{\varepsilon} \equiv-\nabla \cdot\left(k^{\varepsilon}(x) \nabla u_{\varepsilon}(x)\right)=f(x), x \in \Omega . \\
& u_{\varepsilon}=0 \text { on } \partial \Omega,
\end{aligned}
$$

where $\Omega \subset \mathbb{R}^{n}$ is a bounded domain, $k^{\varepsilon}$ is symmetric and satisfies the ellipticity condition $\alpha|\xi|^{2} \leq \xi_{i} k_{i j}^{\varepsilon} \xi_{j} \leq \beta|\xi|^{2}$ for any $\xi \in \mathbb{R}^{n}$ with $0<\alpha \leq \beta$ independent of $\varepsilon$, and $\varepsilon$ is a small positive parameter standing for the scale ratio between the micro structure and the whole domain.

This problem has attracted a great deal of attention because of its relevance to several important practical problems such as flow in porous media and mechanical properties of composite materials. Many numerical homogenization methods have been proposed. We refer to the short reviews in $[16,17]$ and references therein. We will focus on the residual-free bubbles (RFB) finite element method, which was first proposed in [5] for convection-diffusion problems, and then was extended to treat problem (1.1) in [3, 19]. As shown in [3] and [19], the RFB method has a close relation to the multiscale finite element method (MsFEM) [12].

We will see that as a numerical homogenization method for problem (1.1), the coarse scale part of the RFB scheme is actually the same as a simple version of MsFEM if the coefficient tensor $k^{\varepsilon}$ is symmetric. Furthermore, the whole solution of RFB, which is a coarse scale part plus a bubble part, can be obtained from the MsFEM solution by local reconstruction in each finite element. That means over each element, solving the original problem with MsFEM solution as the boundary condition yields the whole RFB solution.

RFB method can also be viewed as an upscaling procedure, since the coarse scale part of RFB scheme implies a closed coarse scale model. So the support of each bubble, which is a whole element, may be regarded as the sampling window. From this point of view, we introduce the technique of adaptive sampling to take the

\footnotetext{
*Received: August 29, 2010; accepted (in revised version): May 23, 2011. Communicated by Pingwen Zhang.

${ }^{\dagger}$ Department of Mathematics, University of Science and Technology of China, Hefei, 230026, China (yutao83@mail.ustc.edu.cn).

${ }^{\ddagger}$ Corresponding author. Department of Mathematics, Soochow University, Suzhou, 215006, China (xyyue@suda.edu.cn).
} 
advantage of scale separation. We may choose bubble functions with much smaller support rather than the whole element to save the computational cost. Then we get a new scheme very similar to the heterogenous multiscale methods (HMM) proposed in [8] and fully analyzed for elliptic homogenization problems in [10]. If the coarse mesh size $h$ is comparable to the small scale size $\varepsilon$, we may choose bubble functions with larger support than the element (so-called over-sampling technique in $[12,11]$ ) in order to achieve more accuracy.

\section{Relations between RFB method and MsFEM}

Let us first introduce the schemes of RFB and MsFEM, then show some observations on the relations between them.

2.1. RFB finite element method. Let $\mathbb{T}_{h}$ be a regular triangulation of $\Omega$ : $\bar{\Omega}=\bigcup_{T \in \mathbb{T}_{h}} T$. Let $V_{C}^{h} \subset H_{0}^{1}(\Omega)$ denote the conforming piecewise linear finite element space on $\mathbb{T}_{h}$. The bubble space is defined by $V_{b}=\prod_{T \in \mathbb{T}_{h}} H_{0}^{1}(T)$. Then the RFB finite element method reads:

$$
\left\{\begin{array}{l}
\text { Find } u_{h}=u_{C}+u_{b} \in V_{h} \equiv V_{C}^{h} \oplus V_{b} \text { such that } \\
a\left(u_{h}, v_{h}\right) \equiv \int_{\Omega} k^{\varepsilon} \nabla u_{h} \cdot \nabla v_{h} d x=\left(f, v_{h}\right), \quad \forall v_{h}=v_{C}+v_{b} \in V_{h} .
\end{array}\right.
$$

This scheme can be split into two parts naturally by choosing test functions at different scales:

$$
\begin{gathered}
\text { Coarse Scale: } a\left(u_{C}+u_{b}, v_{C}\right)=\left(f, v_{C}\right), \quad \forall v_{C} \in V_{C}^{h} . \\
\text { Fine Scale: } a\left(u_{b}, v_{b}\right)=\left(f, v_{b}\right)-a\left(u_{C}, v_{b}\right), \quad \forall v_{b} \in V_{b} .
\end{gathered}
$$

To obtain the completely coarse scale model, one needs to solve the fine scale equation (2.3) for the bubble part $u_{b}$, and substitute it into the coarse scale equation (2.2). For each element $T \in \mathbb{T}_{h}$, the fine scale equation (2.3) is equivalent to the following local problem:

$$
\begin{aligned}
\mathbb{L}\left(u_{C}+u_{b}\right)=f, & \text { in } T, \\
u_{b}=0, & \text { on } \partial T,
\end{aligned}
$$

i.e. the solution $u_{h}=u_{C}+u_{b}$ is residual free inside each element $T \in \mathbb{T}_{h}$.

2.2. A simple version of MsFEM. The basic idea of MsFEM [12] is to construct the multiscale basis functions instead of the piecewise linear basis functions in standard FEM. A similar idea was also used in [2] where a framework of the generalized finite element method was developed for one-dimensional problems. For each triangle element $T \in \mathbb{T}_{h}$, the standard linear finite element space $V_{C}^{T}=\operatorname{span}\left\{\varphi_{1}, \varphi_{2}, \varphi_{3}\right\}$ is replaced by $V_{M S}^{T}=\operatorname{span}\left\{\psi_{1}, \psi_{2}, \psi_{3}\right\}$ such that

$$
\begin{aligned}
& -\nabla \cdot\left(k^{\varepsilon}(x) \nabla \psi_{j}(x)\right)=0, \text { in } T ; \\
& \psi_{j}=\varphi_{j}, \quad \text { on } \partial T,
\end{aligned}
$$

where $\varphi_{j}$ is the conforming piecewise linear nodal basis at vertex $\mathbf{x}_{j}$. Please note that the multiscale basis functions can be constructed in some other ways; see [12] for more details. With these element basis functions, the global multiscale basis functions can be well-defined, so we get the multiscale finite element space $V_{M S} \subset H_{0}^{1}(\Omega)$. Then a 
conforming multiscale finite element scheme can be defined as: Find $u_{h}^{\varepsilon} \in V_{M S}$ such that

$$
a\left(u_{h}^{\varepsilon}, v_{h}\right) \equiv\left(k^{\varepsilon} \nabla u_{h}^{\varepsilon}, \nabla v_{h}\right)=\left(f, v_{h}\right), \quad \forall v_{h} \in V_{M S} .
$$

For periodic media, i.e.

$$
k^{\varepsilon}(x)=k(x, x / \varepsilon) \text { and } k(x, y) \text { is periodic with respect to } y \in Y \equiv[0,1]^{n},
$$

based on the theory of homogenization, the following error estimate was given in [13]:

$$
\left\|u_{\varepsilon}-u_{h}^{\varepsilon}\right\|_{1} \leq C(h+\sqrt{\varepsilon}+\sqrt{\varepsilon / h}),
$$

where, as in the whole paper, $C>0$ is a generic constant independent of $\varepsilon$ and $h$.

REMARK 2.1. Note that in (2.5), the multiscale basis functions are only linear on each element interface, meanwhile the exact solution is oscillated everywhere in the whole domain. This kind of mismatching leads to the 'poor' term $\sqrt{\varepsilon / h}$ in the error estimate (2.8). This term can be improved to $\varepsilon / h$ by the technique of over-sampling in [11].

2.3. Relations between the two methods. For the MsFEM solution $u_{h}^{\varepsilon} \in$ $V_{M S}$, from the definition of the basis functions (2.5), we have over each element $T \in \mathbb{T}_{h}$,

$$
\mathbb{L} u_{h}^{\varepsilon}=0, \text { in } T \text {. }
$$

For RFB solution $u_{h}=u_{C}+u_{b}$, we have

$$
\mathbb{L}\left(u_{C}+u_{b}\right)=f, \quad \text { in } T .
$$

So at first glance, they are obviously two different methods with some similar features. See [19] for the discussions on the relations between these two schemes. The conclusion was that RFB was closely related to MsFEM.

Actually, if we split the bubble into two parts $u_{b}=\mathbb{M}^{0}\left(u_{C}\right)+\mathbb{M}(f)$, such that

$$
\begin{aligned}
\mathbb{M}^{0}\left(u_{C}\right) \in V_{b}, a\left(u_{C}+\mathbb{M}^{0}\left(u_{C}\right), v\right) & =0, \forall v \in V_{b}, \\
\mathbb{M}(f) \in V_{b}, \quad a(\mathbb{M}(f), v) & =(f, v), \forall v \in V_{b},
\end{aligned}
$$

we will see the key fact that the effect of $\mathbb{M}(f)$ can be canceled in the coarse scale model. Recall the RFB FEM scheme

$$
\begin{aligned}
& a\left(u_{C}+\mathbb{M}^{0}\left(u_{C}\right)+\mathbb{M}(f), v_{C}\right)=\left(f, v_{C}\right), \quad \forall v_{C} \in V_{C}^{h}, \\
& a\left(u_{C}+\mathbb{M}^{0}\left(u_{C}\right)+\mathbb{M}(f), v_{b}\right)=\left(f, v_{b}\right), \quad \forall v_{b} \in V_{b} .
\end{aligned}
$$

Setting $v_{b}=\mathbb{M}^{0}\left(v_{C}\right)$ in the second formula, then adding them up, we have

$$
a\left(u_{C}+\mathbb{M}^{0}\left(u_{C}\right)+\mathbb{M}(f), v_{C}+\mathbb{M}^{0}\left(v_{C}\right)\right)=\left(f, v_{C}+\mathbb{M}^{0}\left(v_{C}\right)\right), \quad \forall v_{C} \in V_{C}^{h} .
$$

Noting that the coefficient tensor $k^{\varepsilon}$ is symmetric, we have

$$
a\left(\mathbb{M}(f), v_{C}+\mathbb{M}^{0}\left(v_{C}\right)\right)=a\left(v_{C}+\mathbb{M}^{0}\left(v_{C}\right), \mathbb{M}(f)\right)=0
$$

by the definition of the operator $\mathbb{M}^{0}$ in $(2.9)$. 
The coarse scale model for RFB The coarse scale model for RFB now reads: Find $u_{C} \in V_{C}^{h}$, such that

$$
a\left(u_{C}+\mathbb{M}^{0}\left(u_{C}\right), v_{C}+\mathbb{M}^{0}\left(v_{C}\right)\right)=\left(f, v_{C}+\mathbb{M}^{0}\left(v_{C}\right)\right), \quad \forall v_{C} \in V_{C}^{h} .
$$

If we define the reconstruction operator by

$$
\mathcal{R}\left(v_{C}\right) \equiv v_{C}+\mathbb{M}^{0}\left(v_{C}\right), \forall v_{C} \in V_{C}^{h}
$$

it is obvious that the multiscale basis functions $\psi_{j}$ in (2.5) satisfy $\psi_{j}=\mathcal{R}\left(\phi_{j}\right)$. So the coarse scale model of RFB (2.11) can be rewritten as: Find $u_{C} \in V_{C}^{h}$, such that

$$
a\left(\mathcal{R}\left(u_{C}\right), \mathcal{R}\left(v_{C}\right)\right)=\left(f, \mathcal{R}\left(v_{C}\right)\right), \quad \forall v_{C} \in V_{C}^{h},
$$

or equivalently: Find $\tilde{u}_{h} \in \mathcal{R}\left(V_{C}^{h}\right)$ such that

$$
a\left(\tilde{u}_{h}, \tilde{v}_{h}\right)=\left(f, \tilde{v}_{h}\right), \quad \forall \tilde{v}_{h} \in \mathcal{R}\left(V_{C}^{h}\right) .
$$

The above scheme is the same as the scheme of MsFEM (2.6) since $V_{M S}=\mathcal{R}\left(V_{C}^{h}\right)$.

So far we have, for the RFB solution (2.6),

$$
u_{h}=u_{C}+\mathbb{M}^{0}\left(u_{C}\right)+\mathbb{M}(f)=u_{h}^{\varepsilon}+\mathbb{M}(f),
$$

where $u_{h}^{\varepsilon}$ is the MsFEM solution. From the error estimate (2.8), we know that $u_{C}+\mathbb{M}^{0}\left(u_{C}\right)=u_{h}^{\varepsilon}$ is a good approximation of $u_{\varepsilon}$. The following setting shows us the effect of $\mathbb{M}(f)$ : For each $T \in \mathbb{T}_{h}$,

$$
\begin{array}{ll}
\mathbb{L} u_{h}=f, & \text { in } T, \\
u_{h}=u_{h}^{\varepsilon}, & \text { on } \partial T .
\end{array}
$$

This means that the RFB solution can be obtained from the MsFEM solution by local reconstruction.

Now we come to the following main result.

THEOREM 2.2. Under the assumption that the coefficient $k^{\varepsilon}$ is symmetric in (1.1), let $u_{h}=u_{C}+\mathbb{M}^{0}\left(u_{C}\right)+\mathbb{M}(f)$ be the solution of RFB scheme (2.1) and $u_{h}^{\varepsilon}$ be the solution of MsFEM (2.6). Then we have

$$
u_{h}^{\varepsilon}=u_{C}+\mathbb{M}^{0}\left(u_{C}\right),
$$

and the induced coarse scale model of RFB scheme (2.1) is equivalent to the MsFEM (2.6). Furthermore, the RFB solution $u_{h}$ can be obtained from the MsFEM solution $u_{h}^{\varepsilon}$ by the local reconstruction (2.15).

REMARK 2.3. Local reconstruction is a common strategy for multiscale simulation; one first solves the macro model on the coarse scale mesh, then solves the original problems locally on the fine scale, over some local regions where the details in fine scale are interesting, using the coarse scale solution as the boundary condition [18].

REMARK 2.4. Based on the variational multiscale method (VMS) [14, 15], an adaptive multiscale methods was developed in [17], where it was pointed out that the coarse scale model of VMS also had a form similar to (2.11) for general symmetric bilinear form $a(\cdot, \cdot)$. 


\section{Adaptive sampling}

RFB method can be regraded as an up-scaling procedure [19]. From this point of view, the support of the bubble can be regarded as the sampling window. One takes the sample, then 'measures' the homogenized (effective) coefficient to complete the modeling on the macroscopic scale. How large a sample one should choose is problem dependent. That is the so-called adaptive sampling.

Let us revisit the RFB method. The coarse scale model of RFB is as follows: Find $u_{C} \in V_{C}^{h}$, such that

$$
a\left(\mathcal{R}\left(u_{C}\right), \mathcal{R}\left(v_{C}\right)\right)=\left(f, \mathcal{R}\left(v_{C}\right)\right), \quad \forall v_{C} \in V_{C}^{h} .
$$

It can be rewritten as

$$
\sum_{T} \int_{T} K_{h}^{*} \nabla u_{C} \cdot \nabla v_{C} d x=\int_{\Omega} f \mathcal{R}\left(v_{C}\right) d x, \quad \forall v_{C} \in V_{C}^{h},
$$

where for each $T \in \mathbb{T}_{H}$,

$$
K_{h}^{*} \nabla u_{C} \cdot \nabla v_{C} \equiv \frac{1}{|T|} \int_{T} k^{\varepsilon} \nabla \mathcal{R}\left(u_{C}\right) \cdot \nabla \mathcal{R}\left(v_{C}\right) d x=\frac{1}{|T|} \int_{T} k^{\varepsilon} \nabla \mathcal{R}\left(u_{C}\right) \cdot \nabla v_{C} d x,
$$

where the second equality is valid thanks to the definitions of the reconstruction operator (2.12) and the bubble function (2.9). Or more precisely, $K_{h}^{*}=\left(\left(K_{h}^{*}\right)_{i j}\right)_{n \times n}$ is defined by

$$
\left.\left(K_{h}^{*}\right)_{i j}\right|_{T}=\frac{1}{|T|} \int_{T} k^{\varepsilon} \nabla \mathcal{R}\left(x_{i}\right) \cdot \nabla x_{j} d x=\mathbf{e}_{j} \cdot<k^{\varepsilon} \nabla \mathcal{R}\left(x_{i}\right)>_{T},
$$

where $x_{i}$ is the $i$-th component of $\mathbf{x} \in \mathbb{R}^{\mathbf{n}}$ and $\left\langle v>_{D}=\frac{1}{|D|} \int_{D} v d x\right.$ denotes the average over the region $D$ for any integrated function $v$.

This means that to take the whole element $T$ as the sampling window is to measure the homogenized (or effective) coefficient on macro scale (of size $h$ ). If there exists scale separation, instead of the whole element, one can take a smaller region as the sampling window.

REMARK 3.1. It is easy to check that the effective coefficient defined above in (3.2) satisfies

$$
K_{h}^{*}<\nabla \mathcal{R}\left(v_{C}\right)>_{T}=\left.K_{h}^{*} \nabla v_{C}\right|_{T}=<k^{\varepsilon} \nabla \mathcal{R}\left(v_{C}\right)>_{T}, \forall v_{C} \in V_{C}^{h}, \forall T \in \mathbb{T}_{h} .
$$

This is the key point for model upscaling: one must keep the balance of the mass or energy in macro (left-hand side) and micro (right-hand side) scales.

REMARK 3.2. The same result as (3.2) was obtained in [19] under the assumption that the source term $f$ is piecewise constant on the triangulation $\mathbb{T}_{h}$.

REMARK 3.3. For periodic media (2.7), the modeling error estimate can be found in $[20]$

$$
\max _{x \in T}\left|K^{*}(x)-K_{h}^{*}\right| \leq C\left(\frac{\varepsilon}{h}+h\right), \quad \forall T \in \mathbb{T}_{h}
$$

where $K^{*}(x)$ is the exact homogenized coefficient in homogenization theory. 
3.1. RFB with adaptive sampling. First assume that there exists scale separation in the problem (1.1) and furthermore $h \gg \varepsilon$. We set the bubble space as: $V_{b}=\prod_{T} V_{b}^{T}$, with $V_{b}^{T}=H_{0}^{1}\left(I_{\delta}\right)$ for each element $T$. We may choose the sample $I_{\delta} \subset T$ with size of $\delta$, such that $0<\varepsilon \ll \delta \ll h$. Hereafter we set $I_{\delta}=x_{T} \oplus[-\delta / 2, \delta / 2]^{n}$, where $x_{T}$ is the barycenter of the element $T$.

We can formally define a new RFB finite element method: Find $u_{h}=u_{C}+u_{b} \in$ $V_{h} \equiv V_{C}^{h} \bigoplus V_{b}$ such that

$$
\begin{gathered}
\text { Coarse Scale: } a\left(u_{C}+u_{b}, v_{C}\right)=\left(f, v_{C}\right), \quad \forall v_{C} \in V_{C}^{h} . \\
\text { Fine Scale: } a\left(u_{b}, v_{b}\right)=\left(f, v_{b}\right)-a\left(u_{C}, v_{b}\right), \quad \forall v_{b} \in V_{b} .
\end{gathered}
$$

Over each element $T \in \mathbb{T}_{h}$, the fine scale Equation (3.6) is equivalent to the following local problem:

$$
\begin{aligned}
& \mathbb{L}\left(u_{C}+u_{b}\right)=f, \quad \text { in } I_{\delta} \subset T, \\
& u_{b}=0, \quad \text { on } \partial I_{\delta},
\end{aligned}
$$

i.e. the solution $u_{h}=u_{C}+u_{b}$ is now residual free only inside each sample $I_{\delta} \subset T \in \mathbb{T}_{h}$. Outside the sampling window, we set $u_{b} \equiv 0$, in $T \backslash I_{\delta}, \forall T \in \mathbb{T}_{h}$.

If we split the bubble into two parts $u_{b}=\mathbb{M}_{\delta}^{0}\left(u_{C}\right)+\mathbb{M}_{\delta}(f)$, such that

$$
\begin{aligned}
& \mathbb{M}_{\delta}^{0}\left(u_{C}\right) \in V_{b}, a\left(u_{C}+\mathbb{M}_{\delta}^{0}\left(u_{C}\right), v\right)=0, \forall v \in H_{0}^{1}\left(I_{\delta}\right), \forall T \in \mathbb{T}_{h}, \\
& \mathbb{M}_{\delta}(f) \in V_{b}, a\left(\mathbb{M}_{\delta}(f), v\right)=(f, v), \forall v \in H_{0}^{1}\left(I_{\delta}\right), \forall T \in \mathbb{T}_{h},
\end{aligned}
$$

we will have the coarse scale model of the new RFB scheme: Find $u_{C} \in V_{C}^{h}$ such that

$$
a\left(u_{C}+\mathbb{M}_{\delta}^{0}\left(u_{C}\right), v_{C}+\mathbb{M}_{\delta}^{0}\left(v_{C}\right)\right)=\left(f, v_{C}+\mathbb{M}_{\delta}^{0}\left(v_{C}\right)\right), \quad \forall v_{C} \in V_{C}^{h} .
$$

Thanks to (3.8), the scheme is equivalent to: Find $u_{C} \in V_{C}^{h}$ such that

$$
a\left(u_{C}+\mathbb{M}_{\delta}^{0}\left(u_{C}\right), v_{C}\right)=\left(f, v_{C}+\mathbb{M}_{\delta}^{0}\left(v_{C}\right)\right), \quad \forall v_{C} \in V_{C}^{h},
$$

Or in another form: Find $u_{C} \in V_{C}^{h}$ such that

$$
a^{*}\left(u_{C}, v_{C}\right) \equiv \sum_{T \in \mathbb{T}_{H}} \int_{T} K_{h}^{*} \nabla u_{C} \cdot \nabla v_{C} d x=\left(f, \mathcal{R}\left(v_{C}\right)\right), \quad \forall v_{C} \in V_{C}^{h},
$$

with

$$
\left.\left(K_{h}^{*}\right)_{i j}\right|_{T}=\frac{1}{|T|} \int_{T} k^{\varepsilon} \nabla \mathcal{R}\left(x_{i}\right) \cdot \nabla x_{j} d x,
$$

where in each element $T$, the reconstruction operator is

$$
\mathcal{R}\left(v_{C}\right)=\left\{\begin{array}{ll}
v_{C}, & x \notin I_{\delta}, \\
v_{C}+\mathbb{M}_{\delta}^{0}\left(v_{C}\right), & x \in I_{\delta},
\end{array} \text { for any } v_{C} \in V_{C}^{h} .\right.
$$

Does this still give a 'correct' macro-scale model? Let's check two extreme cases: $I_{\delta}=T$ or $I_{\delta}=\phi$. First let's go back to the RFB (or MsFEM) scheme (2.11) (or (2.13) - (2.14)). In this case, $I_{\delta}=T$, the sample window is the whole element, and we have known that it works well. By the definition of $\mathbb{M}^{0}$ in (2.9), we have

$$
a\left(u_{C}+\mathbb{M}^{0}\left(u_{C}\right), v_{C}\right)=\left(f, v_{C}+\mathbb{M}^{0}\left(v_{C}\right)\right) \quad \forall v_{C} \in V_{C}^{h} .
$$


If we neglect the effect of $\mathbb{M}^{0}\left(u_{C}\right)$ and $\mathbb{M}^{0}\left(v_{C}\right)$, or equivalently set $I_{\delta}=\phi$, we get the standard Galerkin finite element scheme

$$
a\left(u_{C}, v_{C}\right)=\left(f, v_{C}\right) \quad \forall v_{C} \in V_{C}^{h},
$$

and equivalently

$$
\sum_{T} \int_{T} \bar{K}_{h} \nabla u_{C} \cdot \nabla v_{C} d x=\int_{\Omega} f v_{C} d x, \quad \forall v_{C} \in V_{C}^{h}
$$

where for each $T \in \mathbb{T}_{H}$,

$$
\bar{K}_{h} \equiv \frac{1}{|T|} \int_{T} k^{\varepsilon} d x
$$

is the simple arithmetical average. As we have known, for problem (1.1) the standard Galerkin finite element scheme does not work on the coarse mesh with scale of $h>$ $\varepsilon$. So (3.14) is an incorrect coarse scale model. The reason is that although the fine scale bubble $\mathbb{M}^{0}\left(u_{C}\right)$ is negligible when directly compared to the coarse scale function $u_{C}$, this is not the case for $\nabla \mathbb{M}^{0}\left(u_{C}\right)$, which is comparable to $\nabla u_{C}$. On the other hand, if we denote by $a\left(u_{C}+\mathbb{M}^{0}\left(u_{C}\right), v_{C}\right)=\sum_{T} \int_{T}\left(k^{\varepsilon} \nabla u_{C}+k^{\varepsilon} \nabla \mathbb{M}^{0}\left(u_{C}\right)\right) d x$. $\left.\left.\nabla v_{C}\right|_{T} \equiv \sum_{T} \int_{T}\left(\mathbf{q}_{C}+\mathbf{q}_{F}\right) d x \cdot \nabla v_{C}\right|_{T}$, then the macro 'mass' or 'energy' flux $\mathbf{q}_{C}$ and micro flux $\mathbf{q}_{F}$ are comparable. Neglecting of the micro mass flux $\mathbf{q}_{F}$ will lead to the loss of mass balance. For an effective media with coefficient $K^{*}$, we must have the balance (see (3.3))

$$
K^{*}<\nabla u_{\varepsilon}>_{T}=<k^{\varepsilon} \nabla u_{\varepsilon}>_{T}
$$

for any proper micro state $u_{\varepsilon}$. From this point of view, we will see that in the scheme (3.11) - (3.12), only part of micro mass flux on $I_{\delta}$ is included. So we can not expect the balance of mass. We have to modify the bubble functions to keep the balance.

If the size of the sample $I_{\delta}$ is properly chosen, the average flux (or flux density) $<\mathbf{q}_{F}>_{\delta}=\frac{1}{\left|I_{\delta}\right|} \int_{I_{\delta}} k^{\varepsilon} \nabla \mathbb{M}_{\delta}^{0}\left(u_{C}\right) d x$ would be accurate enough. Therefore the modified bubble function $\tilde{\mathbb{M}}_{\delta}^{0}\left(u_{C}\right)$ should satisfy

$$
\frac{1}{|T|} \int_{T} k^{\varepsilon} \nabla \tilde{\mathbb{M}}_{\delta}^{0}\left(u_{C}\right) d x=\frac{1}{\left|I_{\delta}\right|} \int_{I_{\delta}} k^{\varepsilon} \nabla \mathbb{M}_{\delta}^{0}\left(u_{C}\right) d x .
$$

A simple choice is the re-scaled bubble function

$$
\tilde{\mathbb{M}}_{\delta}^{0}\left(v_{C}\right)=\frac{|T|}{\left|I_{\delta}\right|} \mathbb{M}_{\delta}^{0}\left(v_{C}\right) \text {, for any } v_{C} \in V_{C}^{h} .
$$

Then, replacing the bubble functions in the original scheme (3.10), we obtain a new coarse scale model for the adaptive RFB scheme: Find $u_{C} \in V_{C}^{h}$, such that

$$
a\left(u_{C}+\tilde{\mathbb{M}}_{\delta}^{0}\left(u_{C}\right), v_{C}\right)=\left(f, v_{C}+\tilde{\mathbb{M}}_{\delta}^{0}\left(v_{C}\right)\right), \quad \forall v_{C} \in V_{C}^{h} .
$$

Or, equivalently: Find $u_{C} \in V_{C}^{h}$, such that

$$
a^{*}\left(u_{C}, v_{C}\right) \equiv \sum_{T \in \mathbb{T}_{H}} \int_{T} K_{h}^{*} \nabla u_{C} \cdot \nabla v_{C} d x=\left(f, \tilde{\mathcal{R}}\left(v_{C}\right)\right), \quad \forall v_{C} \in V_{C}^{h},
$$


with

$$
\left.\left(K_{h}^{*}\right)_{i j}\right|_{T}=\frac{1}{|T|} \int_{T} k^{\varepsilon} \nabla \tilde{\mathcal{R}}\left(x_{i}\right) \cdot \nabla x_{j} d x
$$

where in each element $T, \tilde{\mathcal{R}}\left(v_{C}\right)=\left\{\begin{array}{ll}v_{C}, & x \notin I_{\delta}, \\ v_{C}+\tilde{\mathbb{M}}_{\delta}^{0}\left(v_{C}\right), & x \in I_{\delta},\end{array}\right.$ for any $v_{C} \in V_{C}^{h}$.

To estimate the modeling error of this scheme, we compare it with the heterogeneous multiscale method (HMM). HMM is a general framework for multiscale problems, proposed in [8] and fully analyzed in [10] for elliptic homogenization problems. We refer to the review paper [9] for the details of using HMM for different classes of multiscale problems. As a numerical homogenization method, HMM has two components: selection of a macroscopic solver and estimating the missing macroscale data by locally solving the fine scale problems.

A simple version of HMM for problem (1.1) [10] has the form as: Find $u_{C} \in V_{C}^{h}$ such that

$$
a_{H M M}^{*}\left(u_{C}, v_{C}\right) \equiv \sum_{T \in \mathbb{T}_{H}} \int_{T} K_{H M M}^{*} \nabla u_{C} \cdot \nabla v_{C} d x=\left(f, v_{C}\right), \quad \forall v_{C} \in V_{C}^{h},
$$

with

$$
\left.\left(K_{H M M}^{*}\right)_{i j}\right|_{T}=\frac{1}{\left|I_{\delta}\right|} \int_{I_{\delta}} k^{\varepsilon} \nabla \mathcal{R}\left(x_{i}\right) \cdot \nabla \mathcal{R}\left(x_{j}\right) d x
$$

where the reconstruction operator $\mathcal{R}$ is defined for any $v_{C} \in V_{C}^{h}$ as

$$
\left\{\begin{array}{c}
-\nabla \cdot\left(k^{\varepsilon} \nabla \mathcal{R}\left(v_{C}\right)\right)=0, \text { in } I_{\delta}, \\
\mathcal{R}\left(v_{C}\right)=v_{C}, \text { on } \partial I_{\delta} .
\end{array}\right.
$$

From (3.8), we readily have $\mathcal{R}\left(v_{C}\right)=v_{C}+\mathbb{M}_{\delta}^{0}\left(v_{C}\right)$. The modeling error for HMM can be bounded for periodic media (2.7) by [10]

$$
|e(H M M)| \equiv \max _{T \in \mathbb{T}_{h}}\left|K^{*}\left(x_{T}\right)-K_{H M M}^{*}\right|_{T} \mid \leq C(\varepsilon / \delta+\delta),
$$

where $K^{*}(x)$ is the exact homogenized coefficient for periodic media and $x_{T}$ is the barycenter of $T \in \mathbb{T}_{h}$.

Remark 3.4. Please note that (3.19) is only a simple version of HMM. If a higher order finite element method is chosen as a macro solver, then at each element $T$, a higher order quadrature rule should be used to compute the element stiffness matrix. In this case, multiple samples may be chosen to support the macro information at the multiple quadrature points for each element $T$ (see [10]). Please also note that HMM has another flexibility in choosing the local micro solver. To define a HMM scheme like (3.19), the key point is to define the local reconstruction operator $\mathcal{R}: V_{C}^{h} \longmapsto H^{1}\left(I_{\delta}\right)$ in (3.21). For HMM, this is only one of the choices. Beside the Dirichlet formulation used here, we may choose periodic boundary conditions, Neumann boundary conditions, or other boundary conditions to define the local reconstruction operator $\mathcal{R}$ with the constraint $\left\langle\nabla \mathcal{R}\left(v_{C}\right)\right\rangle_{I_{\delta}}=\left\langle\nabla v_{C}\right\rangle_{I_{\delta}}$ (see [21]).

The adaptive RFB scheme (3.16) is different with the simple HMM scheme (3.19) in two points: First, their up-scaling procedures (3.18) and (3.20) are slightly different; 
second, they test the right hand side in different ways. More precisely, in the right hand side of (3.19) only coarse scale test functions are used, while in scheme (3.16) multiscale test functions are used. If the right hand side term $f$ does not contain any micro information, by which we mean that either $f$ is independent of $\varepsilon$ or $f \rightarrow f_{0}$ strongly in $H^{-1}$ as $\varepsilon \rightarrow 0$, then there is no need to use the multiscale test functions on the right hand side. But in the case that we only have $f \rightarrow f_{0}$ weakly in $H^{-1}$ as $\varepsilon \rightarrow 0$, the multiscale test functions at the right hand side of (3.16) will play a key role in capturing the micro scale information of the right hand side [7], which we will address more precisely in the following.

Suppose that the coefficient $k^{\varepsilon}=\left(k_{i j}\left(x, \frac{x}{\varepsilon}\right)\right)$ satisfies (2.7) and the source term $f=\nabla \cdot F^{\varepsilon}(x)$ with $F^{\varepsilon}=F(x, x / \varepsilon) \in \mathbb{R}^{n}$ and $F(x, y)$ periodic with respect to $y \in Y \equiv$ $[0,1]^{n}$, then the homogenized equation of (1.1) has the same form as ([7])

$$
\begin{cases}-\nabla \cdot\left(K^{*}(x) \nabla u_{0}(x)\right)=\nabla \cdot F^{*}(x), & x \in \Omega \\ u_{0}(x)=0, & x \in \partial \Omega\end{cases}
$$

where the homogenized coefficient $K^{*}(x)$ is defined by

$$
K_{i j}^{*}(x)=\frac{1}{|Y|} \int_{Y} k_{i m}(x, y)\left(\delta_{m j}+\frac{\partial \chi^{j}}{\partial y_{m}}(x, y)\right) d y,
$$

with $\chi^{j}(x, y)$ being the periodic solution of the following cell problem:

$$
-\nabla_{y} \cdot\left(k(x, y) \nabla_{y} \chi^{j}(x, y)\right)=\nabla_{y} \cdot\left(k(x, y) e_{j}\right),
$$

with zero mean, i.e., $\int_{Y} \chi^{j} d y=0$, and $e_{j}$ is the unit vector in the $j$ th direction.

The homogenized right hand side function $F^{*}(x)$ is defined by

$$
F_{i}^{*}(x)=\frac{1}{|Y|} \int_{Y}\left(F_{i}(x, y)+k_{i j}(x, y) \frac{\partial w}{\partial y_{j}}(x, y)\right) d y,
$$

where $w(x, y)$ is the periodic solution of

$$
-\nabla_{y} \cdot\left(k(x, y) \nabla_{y} w(x, y)\right)=\nabla_{y} \cdot F(x, y),
$$

with zero mean, i.e., $\int_{Y} w d y=0$.

From (3.25) and (3.27), we have

$$
\begin{aligned}
\int_{Y} k(x, y) e_{j} \cdot \nabla_{y} w(x, y) d y & =-\int_{Y} k(x, y) \nabla_{y} \chi(x, y) \cdot \nabla_{y} w(x, y) d y \\
& =\int_{Y} F(x, y) \cdot \nabla_{y} \chi^{j}(x, y) d y
\end{aligned}
$$

Then the homogenized right hand side term has the form

$$
F_{i}^{*}(x)=\frac{1}{|Y|} \int_{Y} F(x, y) \cdot\left(e_{i}+\nabla_{y} \chi^{i}(x, y)\right) d y .
$$

This means that we can obtain the homogenized coefficients $K^{*}$ and $F^{*}$ together by only solving the cell problem (3.25), so there is no need to solve the cell problem (3.27). 
Now the coarse scale model of the adaptive RFB (3.17) can be rewritten as: Find $u_{C} \in V_{C}^{h}$, such that

$$
a^{*}\left(u_{C}, v_{C}\right) \equiv \sum_{T \in \mathbb{T}_{H}} \int_{T} K_{h}^{*} \nabla u_{C} \cdot \nabla v_{C} d x=\sum_{T \in \mathbb{T}_{H}} \int_{T} F_{h}^{*} \cdot \nabla v_{C} d x, \quad \forall v_{C} \in V_{C}^{h},
$$

with $K_{h}^{*}$ being defined in (3.18) and $F_{h}^{*}$ being defined over each element $T \in \mathbb{T}_{H}$ by

$$
\left(F_{h}^{*}\right)_{i} \equiv \frac{1}{|T|} \int_{T} F\left(x, \frac{x}{\varepsilon}\right) \cdot \nabla \tilde{\mathcal{R}}\left(x_{i}\right) d x .
$$

The following theorem is about the modeling error of the adaptive RFB scheme.

THEOREM 3.5. Suppose that in the model problem (1.1),

1. $k^{\varepsilon}$ is symmetric and satisfies the ellipticity condition $\alpha|\xi|^{2} \leq \xi_{i} k_{i j}^{\varepsilon} \xi_{j} \leq \beta|\xi|^{2}$ for any $\xi \in \mathbb{R}^{n}$ with $0<\alpha \leq \beta$ independent of $\varepsilon$;

2. $k^{\varepsilon}=k(x, x / \varepsilon)$ and the source term $f=\nabla \cdot F(x, x / \varepsilon)$ satisfy that both $k(x, y)$ and $F(x, y)$ are smooth with respect to $x$ and periodic with respect to $y \in Y \equiv$ $[0,1]^{n}$;

3. $F$ is uniformly bounded with respect to $\varepsilon$,

then for the coarse scale model of the adaptive RFB scheme (3.16) or (3.29), the modeling error can be bounded by

$$
\left|K_{h}^{*}-K^{*}\left(x_{T}\right)\right|+\left|F_{h}^{*}-F^{*}\left(x_{T}\right)\right| \leq C(\varepsilon / h+\varepsilon / \delta+h+\delta), \forall T \in \mathbb{T}_{h} .
$$

If $u_{C}$ is the solution of the adaptive RFB scheme (3.29) and $u_{0}$ the solution of the homogenized equation (3.23), then

$$
\left\|u_{C}-u_{0}\right\|_{1} \leq C(h+\delta+\varepsilon / h+\varepsilon / \delta) .
$$

Proof. We only prove the modeling error (3.31), since the $H^{1}$-error estimate in (3.32) is a direct consequence of the modeling error. To estimate the first term in (3.31), we first compare $K_{h}^{*}$ with $K_{H M M}^{*}$ in $(3.20)$. Due to (3.21), we can rewrite (3.20) in the following form:

$$
\left(K_{H M M}^{*}\right)_{i j}=\frac{1}{\left|I_{\delta}\right|} \int_{I_{\delta}} k^{\varepsilon} \nabla \mathcal{R}\left(x_{i}\right) \cdot \nabla x_{j} d x, \quad \forall T \in \mathbb{T}_{h}
$$

We know that $\mathcal{R}\left(x_{i}\right)=x_{i}+\mathbb{M}_{\delta}^{0}\left(x_{i}\right)$, so it follows that over each element $T \in \mathbb{T}_{h}$,

$$
\left|K_{h}^{*}-K_{H M M}^{*}\right|=\left|<k^{\varepsilon}>_{T}-<k^{\varepsilon}>_{I_{\delta}}\right| \leq C(\varepsilon / h+\varepsilon / \delta+h),
$$

since $k^{\varepsilon}=k(x, x / \varepsilon)$ and $k(x, y)$ is smooth with respect to $x$ and periodic with respect to $y$. Hence, from the modeling error of HMM (3.22), we have

$$
\left|K_{h}^{*}-K^{*}\left(x_{T}\right)\right| \leq C(\varepsilon / h+\varepsilon / \delta+\delta+h) .
$$

To estimate the second term in (3.31), we introduce two new right hand side functions $\hat{F}_{h}^{*}$ and $\bar{F}_{h}^{*}$, which are obtained by fixing the macro-scale variable at $x_{T}$ in the original multiscale data over each element $T \in \mathbb{T}_{h}$, as

$$
\begin{aligned}
\left(\hat{F}_{h}^{*}\right)_{i} & \equiv \frac{1}{|T|} \int_{T} F\left(x_{T}, x / \varepsilon\right) \cdot \nabla \hat{\mathcal{R}}\left(x_{i}\right) d x, \\
\left(\bar{F}_{h}^{*}\right)_{i} & \equiv \frac{1}{\left|I_{\delta}\right|} \int_{I_{\delta}} F\left(x_{T}, x / \varepsilon\right) \cdot \nabla \overline{\mathcal{R}}\left(x_{i}\right) d x,
\end{aligned}
$$


where for each $v_{C} \in V_{h}, \hat{\mathcal{R}}\left(v_{C}\right)=\left\{\begin{array}{ll}v_{C}, & x \notin I_{\delta} \\ v_{C}+\hat{\mathbb{M}}_{\delta}^{0}\left(v_{C}\right), & x \in I_{\delta}\end{array}\right.$ and $\overline{\mathcal{R}}\left(v_{C}\right)=v_{C}+\overline{\mathbb{M}}_{\delta}^{0}\left(v_{C}\right)$ with the re-scaled bubble function $\hat{\mathbb{M}}_{\delta}^{0}\left(v_{C}\right)=\frac{|T|}{\left|I_{\delta}\right|} \overline{\mathbb{M}}_{\delta}^{0}\left(v_{C}\right)$. Here the bubble function $\overline{\mathbb{M}}_{\delta}^{0}\left(v_{C}\right)$ is also obtained by fixing the macro-scale variable at $x_{T}$ in the original multiscale data in (3.8), i.e.

$$
\int_{I_{\delta}} k\left(x_{T}, x / \varepsilon\right) \nabla\left(v_{C}+\overline{\mathbb{M}}_{\delta}^{0}\left(v_{C}\right)\right) \cdot \nabla v d x=0, \forall v \in H_{0}^{1}\left(I_{\delta}\right), T \in \mathbb{T}_{h}
$$

By the assumption that both $k(x, y)$ and $F(x, y)$ are smooth with respect to $x$, we can prove that

$$
\left\|\nabla\left(\overline{\mathbb{M}}_{\delta}^{0}\left(v_{C}\right)-\mathbb{M}_{\delta}^{0}\left(v_{C}\right)\right)\right\|_{0, I_{\delta}} \leq C \delta\left\|\nabla v_{C}\right\|_{0, I_{\delta}},
$$

and hence over each element $T \in \mathbb{T}_{h}$,

$$
\begin{aligned}
& \left|F_{h}^{*}-\hat{F}_{h}^{*}\right| \leq C(h+\delta), \\
& \left|\hat{F}_{h}^{*}-\bar{F}_{h}^{*}\right|=\left|\left\langle F\left(x_{T}, x / \varepsilon\right)\right\rangle_{T}-\left\langle F\left(x_{T}, x / \varepsilon\right)\right\rangle_{I_{\delta}}\right| \leq C\left(\frac{\varepsilon}{h}+\frac{\varepsilon}{\delta}\right) .
\end{aligned}
$$

Now to complete the proof, we only need to estimate $\left|\bar{F}_{h}^{*}-F^{*}\left(x_{T}\right)\right|$. The argument is similar to that in [6] for up-scaling the velocity fields of multiscale convection-diffusion problems.

From (3.25) and (3.38), it is easy to check that

$$
\overline{\mathcal{R}}\left(x_{i}\right)=x_{i}+\overline{\mathbb{M}}_{\delta}^{0}\left(x_{i}\right)=x_{i}+\varepsilon \chi^{i}\left(x_{T}, x / \varepsilon\right)-\varepsilon \theta_{i}^{\varepsilon},
$$

where $\theta_{i}^{\varepsilon}$ is the boundary corrector, satisfying

$$
\left\{\begin{array}{cc}
-\nabla \cdot\left(k\left(x_{T}, x / \varepsilon\right) \nabla \theta_{i}^{\varepsilon}\right)=0, & \text { in } I_{\delta} \\
\theta_{i}^{\varepsilon}=\chi^{i}\left(x_{T}, x / \varepsilon\right), & \text { on } \partial I_{\delta}
\end{array}\right.
$$

and (see [13])

$$
\left\|\nabla \theta_{i}^{\varepsilon}\right\|_{0, I_{\delta}} \leq C \delta^{(n-1) / 2} \varepsilon^{-1 / 2} .
$$

Then we have, by (3.37), that

$$
\left(\bar{F}_{h}^{*}\right)_{i}=\frac{1}{\left|I_{\delta}\right|} \int_{I_{\delta}} F\left(x_{T}, x / \varepsilon\right) \cdot\left(e_{i}+\varepsilon \nabla_{x} \chi^{i}\left(x_{T}, x / \varepsilon\right)-\varepsilon \nabla \theta_{i}^{\varepsilon}\right) d x .
$$

From (3.28) and (3.44), we have

$$
\begin{aligned}
F_{i}^{*}\left(x_{T}\right)-\left(\bar{F}_{h}^{*}\right)_{i}= & \left(\frac{1}{|Y|} \int_{Y} F\left(x_{T}, y\right) \cdot\left(e_{i}+\nabla_{y} \chi^{i}\left(x_{T}, y\right)\right) d y\right. \\
& \left.-\frac{1}{\left|I_{\delta}\right|} \int_{I_{\delta}} F\left(x_{T}, x / \varepsilon\right) \cdot\left(e_{i}+\varepsilon \nabla_{x} \chi^{i}\left(x_{T}, x / \varepsilon\right)\right) d x\right) \\
& +\frac{\varepsilon}{\left|I_{\delta}\right|} \int_{I_{\delta}} F\left(x_{T}, x / \varepsilon\right) \cdot \nabla \theta_{i}^{\varepsilon} d x \\
:= & I_{1}+I_{2} .
\end{aligned}
$$


The first term can be bounded by $\left|I_{1}\right| \leq C \varepsilon / \delta$. To estimate the second term $I_{2}$, we introduce an auxiliary problem. Let $\phi^{\varepsilon}$ be the solution of the problem

$$
\left\{\begin{array}{cc}
-\nabla \cdot\left(k\left(x_{T}, x / \varepsilon\right) \nabla \phi^{\varepsilon}\right)=\nabla \cdot F\left(x_{T}, x / \varepsilon\right), & \text { in } I_{\delta}, \\
\phi^{\varepsilon}=0, & \text { on } \partial I_{\delta} .
\end{array}\right.
$$

It is easy to check that

$$
\phi^{\varepsilon}=\varepsilon w\left(x_{T}, x / \varepsilon\right)-\varepsilon \theta_{w}^{\varepsilon},
$$

where $\theta_{w}^{\varepsilon}$ is the boundary corrector satisfying

$$
\left\{\begin{array}{cc}
-\nabla \cdot\left(k\left(x_{T}, x / \varepsilon\right) \nabla \theta_{w}^{\varepsilon}\right)=0, & \text { in } I_{\delta} \\
\theta_{w}^{\varepsilon}=w\left(x_{T}, x / \varepsilon\right), & \text { on } \partial I_{\delta}
\end{array}\right.
$$

and

$$
\left\|\nabla \theta_{w}^{\varepsilon}\right\|_{0, I_{\delta}} \leq C \delta^{(n-1) / 2} \varepsilon^{-1 / 2}
$$

By (3.42), we have

$$
\int_{I_{\delta}} k\left(x_{T}, x / \varepsilon\right) \nabla \phi^{\varepsilon} \cdot \nabla \theta_{i}^{\varepsilon} d x=0 .
$$

After substituting (3.46) into the above equality, we obtain

$$
\int_{I_{\delta}} k\left(x_{T}, x / \varepsilon\right) \nabla_{y} w\left(x_{T}, x / \varepsilon\right) \cdot \nabla \theta_{i}^{\varepsilon} d x-\varepsilon \int_{I_{\delta}} k\left(x_{T}, x / \varepsilon\right) \nabla \theta_{w}^{\varepsilon} \cdot \nabla \theta_{i}^{\varepsilon} d x=0 .
$$

Thus,

$$
\begin{aligned}
\int_{I_{\delta}} F\left(x_{T}, x / \varepsilon\right) \cdot \nabla \theta_{i}^{\varepsilon} d x= & \int_{I_{\delta}}\left(F\left(x_{T}, x / \varepsilon\right)+k\left(x_{T}, x / \varepsilon\right) \nabla_{y} w\left(x_{T}, x / \varepsilon\right)\right) \cdot \nabla \theta_{i}^{\varepsilon} d x \\
& -\varepsilon \int_{I_{\delta}} k\left(x_{T}, x / \varepsilon\right) \nabla \theta_{w}^{\varepsilon} \cdot \nabla \theta_{i}^{\varepsilon} d x:=I_{21}+I_{22} .
\end{aligned}
$$

Noting (3.27) and integrating by parts, we obtain

$$
\left|I_{21}\right|=\left|\int_{\partial I_{\delta}}\left(F\left(x_{T}, x / \varepsilon\right)+k\left(x_{T}, x / \varepsilon\right) \nabla_{y} w\left(x_{T}, x / \varepsilon\right)\right) \cdot \mathbf{n} \theta_{i}^{\varepsilon} d s\right| \leq C \delta^{n-1} .
$$

By the estimate (3.43) and (3.48), we have

$$
\left|I_{22}\right| \leq \varepsilon|| \nabla \theta_{i}^{\varepsilon}\left\|_{0, I_{\delta}}|| \nabla \theta_{w}^{\varepsilon}\right\|_{0, I_{\delta}} \leq C \delta^{n-1} .
$$

Therefore, $\left|I_{2}\right| \leq C \varepsilon / \delta$. So we have that, over each element $T \in \mathbb{T}_{h}$,

$$
\left|F_{i}^{*}\left(x_{T}\right)-\left(\bar{F}_{h}^{*}\right)_{i}\right| \leq C \frac{\varepsilon}{\delta} .
$$

Combining the source error terms (3.39), (3.40), and (3.49), we obtain

$$
\left|F_{h}^{*}-F^{*}\left(x_{T}\right)\right| \leq C(\varepsilon / h+\varepsilon / \delta+h+\delta), \forall T \in \mathbb{T}_{h} .
$$

This completes the proof of the theorem. 
3.2. Over-sampling. When the grid size $h$ is comparable to the microscale size $\varepsilon$, the MsFEM (so the RFB) suffers from the 'resonance' error term ' $\varepsilon / h$ ' $[12,11]$. An over-sampling procedure was developed to deal with this difficulty [12]. In the context of bubbles, if we view the support of the bubble function as the sampling window, we can naturally define an over-sampling scheme. We start from the coarse scale model (2.11), and for any $v_{C} \in V_{C}^{h}$, for each element $T \in \mathbb{T}_{h}$, we define a bubble function $\hat{\mathbb{M}}^{0}\left(v_{C}\right)$ over a macro element $S \supset T$ by

$$
\text { Find } \hat{\hat{\mathbb{M}}}^{0}\left(v_{C}\right) \in H_{0}^{1}(S), \quad a\left(v_{C}+\hat{\hat{\mathbb{M}}}^{0}\left(v_{C}\right), v\right)=0, \quad \forall v \in H_{0}^{1}(S),
$$

where $v_{C} \in P_{1}(S)$ is the extension of $v_{C} \in P_{1}(T)$. Then an over-sampling bubble method can be defined as follows: Find $u_{C} \in V_{C}^{h}$, such that for any $v_{C} \in V_{C}^{h}$

$$
\sum_{T \in \mathbb{T}_{h}} \int_{T} k^{\varepsilon} \nabla\left(u_{C}+\hat{\hat{\mathbb{M}}}^{0}\left(u_{C}\right)\right) \cdot \nabla\left(v_{C}+\hat{\hat{\mathbb{M}}}^{0}\left(v_{C}\right)\right) d x=\sum_{T \in \mathbb{T}_{h}} \int_{T} f\left(v_{C}+\hat{\hat{\mathbb{M}}}^{0}\left(v_{C}\right)\right) d x
$$

REMARK 3.6. Though the over-sampling scheme (3.52) is slightly different with the over-sampling MsFEM [12] in formula. They are actually equivalent. An oversampling mixed multiscale finite element [1] was introduced in the same way as what we have done above. However, the scheme (3.52) has been beyond the framework of RFB.

\section{Conclusion}

In this paper, we show that as a numerical homogenization method for problem (1.1), the coarse scale part of the RFB scheme is actually the same as a simple version of MsFEM if the coefficient tensor $k^{\varepsilon}$ is symmetric. The whole solution of RFB can be obtained from the MsFEM solution by local reconstruction in each finite element.

When the RFB method is viewed as an upscaling procedure, the support of each bubble may be regarded as the sampling window. From this point of view, we introduce the technique of adaptive sampling to take the advantage of scale separation. We may choose bubble functions with much smaller support rather than the whole element to save the computational cost. We propose a new adaptive bubble scheme and detailed analysis is presented.

Acknowledgment. The author benefitted a great deal from discussions with Weinan E and Pingbing Ming. This work is supported in part by NSF of China under the Grant 10871190 and by the Qing-Lan Project of Jiangsu Province.

\section{REFERENCES}

[1] T. Arbogast and K. Boyd, Subgrid upscaling and Mixed multiscale finite elements, SIAM J. Numer. Anal., 44, 1150-1171, 2006.

[2] I. Babuska and J. Osborn, Generalized finite element methods: Their performance and their relation to mixed methods, SIAM J. Numer. Anal., 20, 510-536, 1983.

[3] F. Brezzi and L.D. Marini, Augmented spaces, two-level methods, and stabilizing subgrids, Int. J. Numer. Meth. Fluids, 40, 31-46, 2002.

[4] F. Brezzi, D. Marini, and E. Suli, Residual-free bubbles for advection-diffusion problems: The general error analysis, Numer. Math., 85, 31-47, 2000.

[5] F. Brezzi and A. Russo, Choosing bubbles for advection-diffusion problems, Math. Models Methods Appl. Sci., 4, 571-587, 1994.

[6] Z. Chen, W. Deng, and H. Ye, A new upscaling method for the solute transport equations, Disc. Cont. Dyn. Syst. Ser. A, 13(4), 941-960, 2005.

[7] D. Cioranescu and P. Donato, An Introduction to Homogenization, Oxford University Press, New York, 1999. 
[8] W. E and B. Engquist, The heterogeneous multiscale methods, Commun. Math. Sci., 1, 87-132, 2003.

[9] W. E, B. Engquist, X. Li, W. Ren, and E. Vanden-Eijnden, Heterogeneous multiscale methods: A review, Commun. Comput. Phys., 2, 367-450, 2007.

[10] W. E, P. Ming, and P. Zhang, Analysis of the heterogeneous multiscale method for elliptic homogenization problems, J. Amer. Math. Soc., 18, 121-156, 2005.

[11] Y.R. Efendiev, T.Y. Hou, and X.H. Wu, Convergence of a noncomforming multiscale method, SIAM J. Numer. Anal., 37, 888-910, 2000.

[12] T.Y. Hou and X.H. Wu, A multiscale finite element method for elliptic problems in composite materials and porous media, J. Comput. Phys., 134, 169-189, 1997.

[13] T.Y. Hou, X.H. Wu, and Z. Cai, Convergence of a multiscale finite element method for elliptic problems with rapidly oscillating coefficients, Math. Comp., 68, 913-943, 1999.

[14] T.J.R. Hughes, Multiscale phenomena: Green's functions, the Dirichlet-to-Neumann formulation, subgrid scale models, bubbles and the origin of stabilized methods, Comput. Methods Appl. Mech. Engrg., 127, 387-401, 1995.

[15] T.J.R. Hughes, G. Feijoo, L. Mazzei, and J. Quincy, The variational multiscale method - a paradigm for computational mechanics, Comput. Methods Appl. Mech. Engrg., 166, 3-24, 1998.

[16] P. Ming and X. Yue, Numerical methods for multiscale elliptic problems, J. Comput. Phys., 214, 421-445, 2006.

[17] J. Nolen, G. Papanicolaou, and O. Pironneau, A framework for adaptive multiscale methods for elliptic problems, Multi. Model. Simul., 7, 171-196, 2008.

[18] J.T. Oden and K.S. Vemaganti, Estimation of local modelling error and global-oriented adaptive modeling of heterogeneous materials: Error estimates and adaptive algorithms, J. Comp. Phys., 164, 22-47, 2000.

[19] G. Sangalli, Capturing small scales in elliptic problems using a residual-free bubbles finite element method, Multi. Model. Simul., 1, 485-503, 2003.

[20] X. Wu, Y. Efendiev and T. Hou, Analysis of upscaling absolute permeability, Disc. Cont. Dyn. Syst. Ser. B, 2, 185-204, 2002.

[21] X. Yue and W. E, The local microscale problem in the multiscale modeling of strongly heterogeneous media: Effects of boundary conditions and cell size, J. Comput. Phys., 222, 556-572, 2007.

[22] V.V. Zhikov, S.M. Kozlov, and O.A. Oleinik, Homogenization of Differential Operators and Integral Functionals, Springer-verlag Berlin Heidelberg, 1994. 